\title{
Modified Numerals as Post-suppositions
}

\author{
Adrian Brasoveanu* \\ Linguistics, UCSC, 1156 High St., Santa Cruz, CA 95064, abrsvn@gmail.com
}

\begin{abstract}
The paper provides a compositional account of cumulative readings with non-increasing modified numerals (aka van Benthem's puzzle), e.g., Exactly three boys saw exactly five movies. The main proposal is that modified numerals make two kinds of semantic contributions. Their asserted/at-issue contribution is a maximization operator that introduces the maximal set of entities that satisfies their restrictor and nuclear scope. The second contribution is a post-supposition, i.e., a cardinality constraint that needs to be satisfied relative to the context that results after the at-issue meaning is evaluated. We take contexts to be sets of variable assignments relative to which quantificational expressions are interpreted and which are updated as a result of their interpretation.
\end{abstract}

\section{Cumulativity and Modified Numerals}

The goal of the paper is to provide a compositional account of cumulative readings with non-increasing modified numerals (aka van Benthem's puzzle, van Benthem 1986), exemplified in (1) below. We discuss mainly exactly $n$ numerals, but the same problem arises with other non-increasing numerals, e.g., at most $n$.

(1) Exactly three ${ }^{x}$ boys saw exactly five ${ }^{y}$ movies.

The most familiar reading of sentence (1) is the surface-scope distributive one, namely: there are exactly three boys such that each of them saw exactly five movies (possibly different from boy to boy). We are not interested in this reading (although we discuss it briefly later on), but in the cumulative reading, namely: consider the maximal number of boys that saw a movie and the maximal number of movies seen by a boy; there are three such boys and five such movies. This reading of sentence (1) is true in Figure 2 below and false in Figure 1. Note that Figure 1 is exactly like Figure 2, with the addition of boy $\mathbf{b}_{\mathbf{1}}$, movie $\mathbf{m}_{\mathbf{1}}$ and the arrow between them symbolizing the seeing relation at the very top of Figure 1.

Importantly, the cumulative reading is different from: the maximal number of boys that (between them) saw exactly five movies is three. ${ }^{1}$ This is actually

\footnotetext{
* I want to thank Lucas Champollion for mentioning this problem in the context of plural logic and for subsequent discussion and comments, Pranav Anand, Rick Nouwen, Jessica Rett, Livio Robaldo, the UCSC S-Circle audience (11/23/2009) and three anonymous Amsterdam Colloquium 2009 reviewers for comments on earlier versions of this paper and Melissa Frazier and Grant McGuire for data discussion.

1 As Krifka (1999), Landman (2000) and Ferreira (2007) observe. See Robaldo (2009) for a different take on the data.
} 
not a reading of sentence (1), although it bears some resemblance to its distributive reading. In fact, the situations in Figures 1 and 2 above distinguish between them: the cumulative reading is intuitively false in Figure 1 (4 boys and 6 movies) and true in Figure 2, while the other 'reading' just mentioned is true in both situations. The distinction between the cumulative reading and this other 'reading' is important for theoretical reasons: many formal systems derive something like it when they attempt to capture the cumulative reading.

(2)

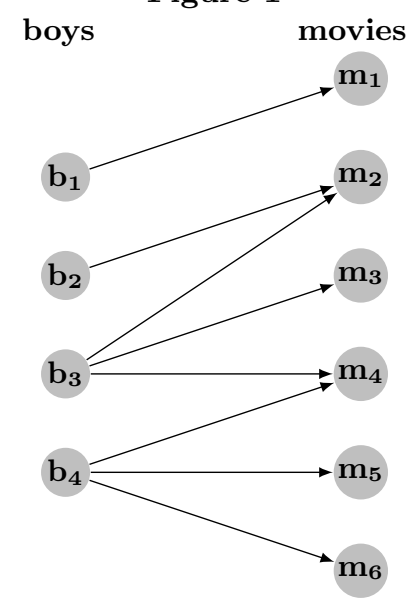

Figure 2

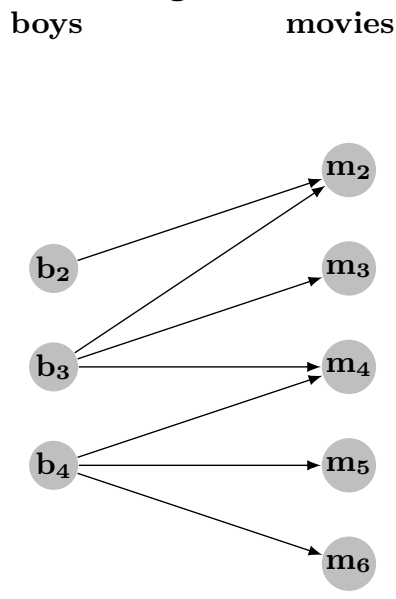

Our proposal is that modified numerals make two kinds of contributions to the meaning of sentences like $(1)$ : $(i)$ their asserted/at-issue contribution is a maximization operator that introduces the maximal set of entities that satisfies their restrictor and nuclear scope; $(i i)$ they also contribute a post-supposition, i.e., a cardinality constraint (e.g., exactly five) that needs to be satisfied relative to the context that results after the at-issue meaning is evaluated. See Lauer (2009) for another use of this notion and Farkas (2002) and Constant (2006) for related post-assertion constraints on output contexts.

For our purposes, contexts are sets of total variable assignments relative to which quantificational expressions are interpreted and which are updated as a result of the interpretation of such expressions. That is, we work with a simplified version of Dynamic Plural Logic (DPIL, van den Berg 1996).

The main difference between the present account and Krifka (1999) is conceptual: we take modified numerals to constrain quantificational - not focus alternatives, where a quantificational alternative is a set of assignments satisfying a quantificational expression. Thus, we reconceptualize DPIL as the logic of quantificational alternatives in natural language interpretation.

\section{Modified Numerals as Post-suppositions}

We work with the usual models for classical first-order logic (FOL) $\mathfrak{M}=\langle\mathfrak{D}, \mathfrak{I}\rangle$ : $\mathfrak{D}$ is the domain of individuals and $\mathfrak{I}$ is the basic interpretation function such 
that $\mathfrak{I}(R) \subseteq \mathfrak{D}^{n}$ for any $n$-ary relation $R$. An $\mathfrak{M}$-assignment $g$ is a total function from the set of variables $\mathcal{V}$ to $\mathfrak{D}$. The essence of quantification in FOL is pointwise/variablewise manipulation of variable assignments, abbreviated $h[x] g$. We generalize this to sets of assignments $H[x] G$ cumulative-quantification style.

(3) $h[x] g:=h$ differs from $g$ at most with respect to the value assigned to $x$ (4) $H[x] G:=\left\{\begin{array}{l}\text { for all } h \in H, \text { there is a } g \in G \text { such that } h[x] g \\ \text { for all } g \in G, \text { there is a } h \in H \text { such that } h[x] g\end{array}\right.$

This is not a way of sneaking cumulativity into the system. Formally, $H[x] G$ is a natural generalization of $h[x] g$ : it is an equivalence relation over sets of total assignments, just as $h[x] g$ is an equivalence relation over total assignments.

Atomic formulas are tests, i.e., they check that the input context $G$ satisfies them and pass this context on. Cardinality constraints on the values of variables are also tests; the cardinality of the set of individuals $X$ is symbolized as $|X|$. Dynamic conjunction and random assignment are interpreted DPL-style.

(7) $\llbracket x=n \rrbracket^{\langle G, H\rangle}=\mathbb{T}$ iff $G=H$ and $|H(x)|=n$

(8) $\llbracket x \leq n \rrbracket^{\langle G, H\rangle}=\mathbb{T}$ iff $G=H$ and $|H(x)| \leq n$

(9) $\llbracket x \geq n \rrbracket^{\langle G, H\rangle}=\mathbb{T}$ iff $G=H$ and $|H(x)| \geq n$

(10) $\llbracket \phi \wedge \psi \rrbracket^{\langle G, H\rangle}=\mathbb{T}$ iff there is a $K$ s.t. $\llbracket \phi \rrbracket^{\langle G, K\rangle}=\mathbb{T}$ and $\llbracket \psi \rrbracket^{\langle K, H\rangle}=\mathbb{T}$

(11) $\llbracket[x] \rrbracket^{\langle G, H\rangle}=\mathbb{T}$ iff $H[x] G$

The translation of singular indefinite articles and bare numerals has the form given in (12) below. Square brackets [] indicate restrictor formulas and round brackets ( ) indicate nuclear scope formulas. As (13) and (14) show, $n$ is 1 for singular indefinite articles, $n$ is 2 for the bare numeral two etc. This translation schema is just an abbreviation, provided in (15). Proper names are interpreted like indefinites; their restrictor requires the variable to have the same value as a non-logical constant, e.g., JASPER in (16) below (where JASPER denotes the individual Jasper). Pronouns are indexed with the variable introduced by their antecedent and their translation is that variable itself; we ignore differences between singular and plural pronouns. For example, (17) is translated as in (18).

(12) $\exists x[x=n \wedge \phi](\psi) \quad$ intuitively: $n \phi$-individuals are $\psi$

(13) $\mathrm{A}^{x}$ wolf came in. $\rightsquigarrow \exists x[x=1 \wedge \operatorname{WOLF}(x)](\operatorname{COME}-\mathrm{IN}(x))$

(14) Two ${ }^{x}$ wolves came in. $\rightsquigarrow \exists x[x=2 \wedge \operatorname{WOLF}(x)](\operatorname{COME}-\mathrm{IN}(x))$

(15) $\exists x[x=n \wedge \phi](\psi):=[x] \wedge x=n \wedge \phi \wedge \psi$

(16) $\exists x[x=\mathrm{JASPER}](\phi):=[x] \wedge x=\mathrm{JASPER} \wedge \phi$

(17) $\mathrm{A}^{x}$ wolf came in. $\mathrm{It}_{x}$ bit Jasper ${ }^{y}$.

(18) a. $\exists x[x=1 \wedge \operatorname{WOLF}(x)](\operatorname{COME}-\operatorname{IN}(x)) \wedge \exists y[y=\operatorname{JASPER}](\operatorname{BiTE}(x, y))$

b. $[x] \wedge x=1 \wedge \operatorname{WOLF}(x) \wedge \operatorname{COME}-\mathrm{IN}(x) \wedge[y] \wedge y=\operatorname{JASPER} \wedge \operatorname{BITE}(x, y)$

The definition of truth below says that a formula $\phi$ is true if there is at least one successful way to update the input context of evaluation $G$ with $\phi$. Except 
for the fact that we work with sets of assignments instead of single assignments, our interpretation function and truth definition are not different from the corresponding FOL or DRT/FCS notions (only their packaging is different).

(19) Truth: a formula $\phi$ is true relative to an input set of assignments $G$ iff there is an output set of assignments $H$ such that $\llbracket \phi \rrbracket^{\langle G, H\rangle}=\mathbb{T}$.

\subsection{Modified Numerals}

We capture the meaning of modified numerals by means of a maximization operator $\mathbf{M}$ that enables us to introduce the set of all individuals satisfying their restrictor and nuclear scope. For example, $\mathbf{M}([x] \wedge \operatorname{BOY}(x))$ introduces the variable $x$ and requires it to store all and only the individuals satisfying $\operatorname{BOY}(x)$ : $[x] \wedge \operatorname{BOY}(x)$ ensures that we store in $x$ only individuals that satisfy $\operatorname{BOY}(x)$ and $\mathbf{M}$ ensures that we cannot store more individuals in $x$ and still satisfy BOY $(x)$. We can now provide a preliminary translation for modified numerals, which has the form in (21). For example, Exactly three ${ }^{x}$ boys left is translated as in (22): we store in $x$ all the boys that left and test that there are 3 such entities.

$$
\begin{aligned}
& \llbracket \mathbf{M}(\phi) \rrbracket^{\langle G, H\rangle}=\mathbb{T} \text { iff } \\
& \llbracket \phi \rrbracket^{\langle G, H\rangle}=\mathbb{T} \text { and there is no } H^{\prime} \text { s.t. } H \subsetneq H^{\prime} \text { and } \llbracket \phi \rrbracket^{\left\langle G, H^{\prime}\right\rangle}=\mathbb{T} \\
& \text { exactly } n \quad \exists x=n[\phi](\psi):=\mathbf{M}([x] \wedge \phi \wedge \psi) \wedge x=n \\
& \exists x=3[\operatorname{Boy}(x)](\operatorname{LEAVE}(x)):=\mathbf{M}([x] \wedge \operatorname{BOY}(x) \wedge \operatorname{LEAVE}(x)) \wedge x=3
\end{aligned}
$$

We can further elaborate on the above sentence with They $y_{x}$ were hungry and derive the intuitively-correct truth conditions for the resulting discourse. But we derive incorrect truth conditions for sentence (1):

$$
\begin{aligned}
& \text { a. } \exists x=3[\operatorname{Boy}(x)](\exists y=5[\operatorname{Movie}(y)](\sec (x, y))) \\
& \text { b. } \mathbf{M}([x] \wedge \operatorname{BOY}(x) \wedge \mathbf{M}([y] \wedge \operatorname{MOvie}(y) \wedge \operatorname{SeE}(x, y)) \wedge y=5) \wedge x=3
\end{aligned}
$$

We do not derive the cumulative reading, true only in Figure 2, but the 'reading' true in both Figure 1 and Figure 2: the maximal number of boys that (between them) saw exactly 5 movies is 3 . What we want is a translation that places the cardinality requirement $y=5$ contributed by the direct object outside the scope of the operator $\mathbf{M}([x] \wedge \ldots)$ contributed by the subject, as shown in (24a) or, equivalently, (24b) below. These formulas capture the cumulative reading of (1): we introduce the maximal set $x$ of boys that saw a movie and the maximal set $y$ of movies seen by a boy and test that there are 5 such movies and 3 such boys.

$$
\begin{array}{ll}
\text { a. } & \mathbf{M}([x] \wedge \operatorname{BOY}(x) \wedge \mathbf{M}([y] \wedge \operatorname{MOvie}(y) \wedge \operatorname{seE}(x, y))) \wedge y=5 \wedge x=3 \\
\text { b. } & \mathbf{M}([x] \wedge \operatorname{BOY}(x) \wedge[y] \wedge \operatorname{MOviE}(y) \wedge \operatorname{SeE}(x, y)) \wedge y=5 \wedge x=3
\end{array}
$$

\subsection{Post-suppositions}

To compositionally derive such a representation, we will take cardinality requirements to be part of a dimension of meaning separate from the asserted/at-issue 
meaning (but closely integrated with it). We take them to be post-suppositions, i.e., tests on output contexts, as opposed to presuppositions, which are tests on input contexts.

Post-suppositions are formulas introduced at certain points in the interpretation that are passed on from local context to local context and that need to be satisfied only globally, relative to the final output context. Our notion of context is now a set of assignments $G$ indexed with a set of tests $\zeta$, represented as $G[\zeta]$. All the above formulas are interpreted in the same way, except that we accumulate post-suppositions as we incrementally update our input context. Thus, our interpretation function is of the form $\llbracket \cdot \rrbracket^{\left\langle G[\zeta], H\left[\zeta^{\prime}\right]\right\rangle}$, where $\zeta \subseteq \zeta^{\prime}$.

We mark a test $\phi$ as a post-supposition by superscripting it, as shown in (25) below; semantically, we completely ignore the input set of assignments $G$ and simply add $\phi$ to the set of tests $\zeta$. Such superscripted tests are post-suppositional in the sense that they are required to be true relative to the final output context. This is formalized by the new definition of truth in (26) below, which treats the formulas $\psi_{1}, \ldots, \psi_{m}$ as post-suppositions because they are tests performed on the final output set of assignments $H$ (again: contrast this with presuppositions).

$$
\llbracket^{\phi} \rrbracket^{\left\langle G[\zeta], H\left[\zeta^{\prime}\right]\right\rangle}=\mathbb{T} \text { iff } \phi \text { is a test, } G=H \text { and } \zeta^{\prime}=\zeta \cup\{\phi\}
$$

Truth: a formula $\phi$ is true relative to an input context $G[\emptyset]$, where $\emptyset$ is the empty set of tests, iff there is an output set of assignments $H$ and a (possibly empty) set of tests $\left\{\psi_{1}, \ldots, \psi_{m}\right\}$ s.t. $\llbracket \phi \rrbracket^{\left\langle G[\emptyset], H\left[\left\{\psi_{1}, \ldots, \psi_{m}\right\}\right]\right\rangle}=\mathbb{T}$

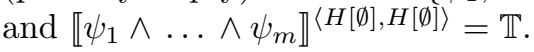

Modified numerals are interpreted as before, except that the cardinality requirement is a post-supposition. Numeral modifiers exactly, at most, at least etc. are functions taking a bare numeral as their argument and introducing $(i)$ a maximization operator $\mathbf{M}$ scoping over the random assignment and the restrictor and nuclear scope formulas and (ii) a post-supposition consisting of the cardinality requirement ordinarily contributed by the bare numeral. The resulting translation of sentence (1) in (28) below derives the correct cumulative truth conditions. Note that the four formulas in $(28 \mathrm{a}, \mathrm{b}, \mathrm{c}, \mathrm{d})$ are truth-conditionally equivalent.

$$
\begin{aligned}
& \text { exactly } n \quad \exists^{x=n}[\phi](\psi):=\mathbf{M}([x] \wedge \phi \wedge \psi) \wedge{ }^{x=n} \\
& \text { a. } \exists^{x=3}[\operatorname{BOY}(x)]\left(\exists^{y=5}[\operatorname{MOviE}(y)](\operatorname{SEE}(x, y))\right) \\
& \text { b. } \mathbf{M}\left([x] \wedge \operatorname{BOY}(x) \wedge \mathbf{M}([y] \wedge \operatorname{MOVIE}(y) \wedge \operatorname{SEE}(x, y)) \wedge{ }^{y=5}\right) \wedge{ }^{x=3} \\
& \text { c. } \mathbf{M}([x] \wedge \operatorname{BOY}(x) \wedge \mathbf{M}([y] \wedge \operatorname{MOVIE}(y) \wedge \operatorname{SEE}(x, y))) \wedge{ }^{y=5} \wedge \wedge^{x=3} \\
& \text { d. } \mathbf{M}([x] \wedge \operatorname{BOY}(x) \wedge[y] \wedge \operatorname{MOVIE}(y) \wedge \operatorname{SEE}(x, y)) \wedge y=5 \wedge x=3
\end{aligned}
$$

Just as before, if we elaborate on (1) with Theyx liked themy, we derive the correct interpretation for the entire discourse: every one of the three boys liked every movie he saw (and not the movies some other boy saw).

The proposed analysis of modified numerals involves three crucial ingredients: ( $i$ ) evaluation pluralities (sets of assignments), (ii) maximization operators over such pluralities and (iii) post-suppositions and their unusual scoping behavior. The following three sections provide independent evidence for each of them. 


\section{Universal Quantifiers}

The fact that we use evaluation pluralities enables us to also account for cumulative readings of universals (Schein 1993, Kratzer 2000, Champollion 2009). ${ }^{2}$

Consider the sentence in (29) below (from Kratzer 2000). Its cumulative reading is: there are three editors such that each of them caught at least one mistake and every mistake was caught by at least one of the three editors. We translate distributive universal quantification as shown in (30): we introduce the set of all individuals $x$ that satisfy the restrictor $\phi$ by means of the maximization operator $\mathbf{M} x$ and we check that each of these individuals also satisfies the nuclear scope $\psi$ by means of the distributivity operator $\delta$.

(29) Three $^{x}$ copy editors (between them) caught every ${ }^{y}$ mistake in the manuscript.

(30) $\forall x[\phi] \delta(\psi):=\mathbf{M} x(\phi) \wedge \delta(\psi)$

$\mathbf{M} x$ is the selective counterpart of the unselective, adverbial $\mathbf{M}$ : unselective $\mathbf{M}$ maximizes over sets of assignments $H$, while selective $\mathbf{M} x$ maximizes over sets of individuals $H(x)$ ('selective' and 'unselective' in the sense of Lewis 1975). Using unselective maximization for modified numerals is justified by the fact that their modifier can be non-adjacent/adverbial, as shown by the examples below.

(31) $\llbracket \mathbf{M} x(\phi) \rrbracket^{\left\langle G[\zeta], H\left[\zeta^{\prime}\right]\right\rangle}=\mathbb{T}$ iff $\llbracket[x] \wedge \phi \rrbracket^{\left\langle G[\zeta], H\left[\zeta^{\prime}\right]\right\rangle}=\mathbb{T}$ and there is no $H^{\prime}$ s.t. $H(x) \subsetneq H^{\prime}(x)$ and $\llbracket[x] \wedge \phi \rrbracket^{\left\langle G[\zeta], H^{\prime}\left[\zeta^{\prime}\right]\right\rangle}=\mathbb{T}$.

(32) Three boys saw five movies, exactly/precisely/at (the) most.

(33) The league limits teams to playing two games in a row - or, at the most, four games in five days, NBA spokesman Tim Frank says. ${ }^{3}$

The distributivity operator $\delta$ in (34) (based on Brasoveanu 2008) says that we distributively update a set of assignments $G$ with a formula $\phi$ by updating each singleton set $\{g\} \subseteq G$ with $\phi$ and taking the union of the resulting output sets of assignments $K$. In addition, $\delta$ discharges all post-suppositions contributed by the formula in its scope. Thus, just like presuppositions, post-suppositions are not always satisfied globally, but can be satisfied/discharged at intermediate points in the semantic composition, i.e., in more local output contexts.

$\llbracket \delta(\phi) \rrbracket^{\left\langle G[\zeta], H\left[\zeta^{\prime}\right]\right\rangle}=\mathbb{T}$ iff $\zeta=\zeta^{\prime}$ and there exists a partial function $\mathcal{F}$ from assignments $g$ to sets of assignments $K$, i.e., of the form $\mathcal{F}(g)=K$, s.t.

(i) $G=\operatorname{Dom}(\mathcal{F})$ and $H=\bigcup \operatorname{Ran}(\mathcal{F})$ and $(i i)$ for all $g \in G$, there is a (possibly empty) set of formulas $\left\{\psi_{1}, \ldots, \psi_{m}\right\}$ s.t.

$\llbracket \phi \rrbracket^{\left\langle\{g\}[\zeta], \mathcal{F}(g)\left[\zeta \cup\left\{\psi_{1}, \ldots, \psi_{m}\right\}\right]\right\rangle}=\mathbb{T}$ and $\llbracket \psi_{1} \wedge \ldots \wedge \psi_{m} \rrbracket^{\langle\mathcal{F}(g)[\zeta], \mathcal{F}(g)[\zeta]\rangle}=\mathbb{T}$

The translation of sentence (29) above is provided below: we introduce a set $x$ of three editors and the set $y$ of all mistakes and check that, for every assignment $h$ in the resulting output state $H$, the editor $h(x)$ caught the mistake $h(y)$.

\footnotetext{
${ }^{2}$ I am indebted to Lucas Champollion for many helpful comments on this subsection.

${ }^{3}$ From the Corpus of Contemporary American English, www.americancorpus.org.
} 

a. $\exists x[x=3 \wedge \operatorname{EDitoR}(x)](\forall y[\operatorname{mistake}(y)] \delta(\operatorname{CATCH}(x, y)))$
b. $[x] \wedge x=3 \wedge \operatorname{EditoR}(x) \wedge \mathbf{M} y(\operatorname{Mistake}(y)) \wedge \delta(\operatorname{CATCH}(x, y))$

The distributivity operator $\delta$ is semantically vacuous in (35) - but not always. Consider the example below, from Kratzer (2000). This sentence does not have a cumulative reading to the effect that, between them, the editors caught a total of 500 mistakes. Its only reading is the distributive one: every editor is such that $\mathrm{s} /$ he caught 500 mistakes. We derive the distributive reading if the universal takes scope over the numeral every $>>500$. That is, cumulative readings are possible with universals only if they have narrow scope relative to the numerals they 'cumulate' with. As long as the non-surface scope $500>>$ every is blocked for sentence (36), we correctly derive the unavailability of the cumulative reading.

(36) Every $^{x}$ copy editor caught $500^{y}$ mistakes in the manuscript.

$$
\forall x[\operatorname{Editor}(x)] \delta(\exists y[y=500 \wedge \operatorname{Mistake}(y)](\mathrm{CATCH}(x, y)))
$$

We also account for mixed cumulative-distributive sentences, e.g., Three video games taught every quarterback two new plays (Schein 1993): every quarterback is related cumulatively to three video games (a total of three video games taught all the quarterbacks), but distributes in the usual way over two new plays (every quarterback learned two possibly different plays). This automatically follows in our system if we preserve the surface-scope relations three $>>$ every $>>$ two.

Finally, we capture the distributive reading of (1) by means of the operator $\delta$ : distributive modified numerals have a $\delta$ operator over their nuclear scope.

$$
\begin{aligned}
& \exists^{x=n}[\phi] \delta(\psi):=\mathbf{M}([x] \wedge \phi \wedge \delta(\psi)) \wedge{ }^{x=n} \\
& \exists^{x=3}[\operatorname{BOY}(x)] \delta\left(\exists^{y=5}[\operatorname{MOviE}(y)] \delta(\operatorname{SEE}(x, y))\right)
\end{aligned}
$$

\section{Implicatures}

Analyzing modified numerals by means of a maximization operator over evaluation pluralities enables us to account for the independent observation that modified numerals do not trigger scalar implicatures, unlike bare numerals/indefinites. This is because the operator $\mathbf{M}$ contributed by modified numerals effectively eliminates referential uncertainty. In any given world, the variable introduced by a modified numeral can be associated with only one set of values: the set of all entities satisfying the restrictor and nuclear scope of the modified numeral. This is shown by the contrast below (from Umbach 2006).

(40) $\{$ Two/\#At least two $\}$ boys were selling coke. They were wearing black jackets. Perhaps there were others also selling coke, but I didn't notice.

If there are more than two boys selling coke, the variable introduced by the bare numeral two can take different sets of two boys as values, i.e., the output contexts obtained after the update with a bare numeral may assign different sets of values to the variable contributed by the bare numeral. In contrast, the variable introduced by at least two has only one possible value: the set of all boys 
selling coke. In any given world, all output contexts obtained after the update with a modified numeral assign the same value to the variable it contributes.

Thus, scalar implicatures are triggered by items that allow for referential indeterminacy/uncertainty. It is this semantic uncertainty that kicks off the pragmatic inferential process resulting in the addition of scalar implicatures.

But referential certainty is distinct from epistemic certainty. Suppose that our contexts are not simply sets of assignments $G, H, \ldots$, but pairs of a world and a set of assignments $\langle w, G\rangle,\left\langle w^{\prime}, H\right\rangle, \ldots$ (in the spirit of Heim 1982). The information state at any point in discourse consists of all the pairs that are still live options, i.e., that are compatible with all the previous updates. Referential uncertainty is encoded by the second member of such pairs. Epistemic uncertainty is encoded by the first member of the pairs, i.e., by the set of worlds in the current information state - aka the current Context Set (Stalnaker 1978).

Modified numerals are referentially determined, but epistemically uncertain. If we fix the world, the variable contributed by the modified numeral has only one value, but this value may vary from world to world. Hence, we can use them (as opposed to their bare counterparts) only if we are epistemically uncertain about the cardinality of the maximal set of entities introduced by them.

This is the reason for the modal readings of indicative sentences with modified numerals (no need for insertion of covert modals, as in Nouwen 2009 and references therein). Jasper invited maximally 50 people to his party (from Nouwen 2009) is felicitous only if the speaker is uncertain with respect to the cardinality of the set of invited people (hence the 'range of values' interpretation). So, if the speaker does not know how many people Jasper invited, it is unacceptable to continue with: 43, to be precise. The same pragmatic infelicity can arise intrasententially: \#A hexagon has at most/maximally/up to 11 sides (Nouwen 2009) is infelicitous if we know what the word hexagon means.

Finally, given their epistemic uncertainty, modified numerals trigger epistemic implicatures of the kind proposed in Büring (2008) for at least.

\section{Modals and Modified Numerals}

This section provides independent evidence for the analysis of modified numerals in terms of post-suppositions. The unusual scoping behavior of post-suppositions and their interaction with distributivity enables us to capture the scopal interactions between modified numerals and modals. This is a novel result that solves an outstanding problem for the current analyses based on standard assumptions about the semantics of minimizers/maximizers and necessity/possibility modals (see Nouwen 2009 and references therein for more discussion). We provide the representations for two typical sentences (from Nouwen 2009) instantiating this problem and leave a more detailed discussion for another occasion.

Necessity modals are analyzed as distributive universal quantifiers in the modal domain; in (42) below, $R$ is a contextually-provided accessibility relation and $R_{w^{*}}(w)$ is interpreted as: $w$ is an $R$-accessible world from the actual world $w^{*}$. The reading of sentence (41) we are after is: the minimum number of books 
that Jasper is allowed to read is 10. The update in (43) captures this reading: each world $w$ that is $R$-accessible from the actual world $w^{*}$ is such that, if we store in $y$ all the books Jasper read, the cardinality of the set of books is at least 10. That is: Jasper reads at least 10 books in every deontically-ideal world $w$.

(41) Jasper ${ }^{x}$ should $^{w}$ read at least ten ${ }^{y}$ books (to please his mother).

(42) $\mathbf{N E C} w(\phi):=\mathbf{M}\left([w] \wedge R_{w^{*}}(w)\right) \wedge \delta(\phi)$

(43) a. $\mathbf{N E C} w\left(\exists x[x=\mathrm{JASPER}]\left(\exists^{y \geq_{w} 10}\left[\operatorname{BOOK}_{w}(y)\right]\left(\operatorname{READ}_{w}(x, y)\right)\right)\right)$

b. $\mathbf{M}\left([w] \wedge R_{w^{*}}(w)\right) \wedge$

$$
\delta\left([x] \wedge x=\operatorname{JASPER} \wedge \mathbf{M}\left([y] \wedge \operatorname{BOOK}_{w}(y) \wedge \operatorname{READ}_{w}(x, y)\right) \wedge y \geq{ }_{w} 10\right)
$$

We also account for maximal permissions like (44) below, interpreted as: the maximum number of people Jasper is allowed to invite is 10 . We take possibility modals to be the counterparts of a modified numeral in the modal domain that contributes a non-singleton cardinality requirement. The maximization operator M over worlds is independently justified by modal subordination (Roberts 1989), e.g., A wolf might come in. It would eat Jasper first is interpreted as: for any epistemic possibility of a wolf coming in, the wolf eats Jasper first. The update in (46) introduces all the worlds $w$ that are $R$-accessible from the actual world $w^{*}$ and such that Jasper invites some people in $w$. For each such world $w, y$ stores all the people invited by Jasper. Finally, we check that there is more than one world $w$ and that the cardinality of the set $y$ in each world $w$ is at most 10 .

(44) Jasper $^{x}$ is allowed ${ }^{w}$ to invite at most ten ${ }^{y}$ people.

(45) $\quad \operatorname{POS} w(\phi):=\exists^{w>1}\left[R_{w^{*}}(w)\right](\phi)=\mathbf{M}\left([w] \wedge R_{w^{*}}(w) \wedge \phi\right) \wedge w>1$

(46) a. $\operatorname{POS} w\left(\exists x[x=\mathrm{JASPER}]\left(\exists^{y \leq w} 10\left[\operatorname{PERSON}_{w}(y)\right]\left(\operatorname{INVITE}_{w}(x, y)\right)\right)\right)$ b. $\underset{y \leq w 10}{\mathbf{M}}\left([w] \wedge R_{w^{*}}(w) \wedge[x] \wedge x=\operatorname{JASPER}_{y>1} \wedge[y] \wedge \operatorname{PERSON}_{w}(y) \wedge \operatorname{INVITE}_{w}(x, y)\right) \wedge$

\section{Conclusion}

We introduced a framework that distinguishes evaluation plurality (sets of assignments) from domain plurality (non-atomic individuals). The maximization operator $\mathbf{M}$ and the distributivity operator $\delta$ are to evaluation pluralities what the familiar Link-style sum and distributivity operators are to domain pluralities. Cumulativity is just non-distributivity with respect to evaluation pluralities, while collectivity is just non-distributivity with respect to domain pluralities.

Modified numerals are maximal and introduce cardinality post-suppositions, which are constraints on output contexts - in contrast to presuppositions, which constrain input contexts. Just as presuppositions, post-suppositions can be satisfied/discharged non-globally, e.g., in the scope of distributivity operators. Postsuppositions are distinct from regular at-issue meaning with respect to their evaluation order: they can constrain the final, global output context. The exceptional scoping behavior of post-suppositions enables us to account for cumulative readings of non-increasing modified numerals and for their interaction with modal verbs. The referential maximality of modified numerals accounts for the fact that they do not trigger scalar implicatures, but only epistemic implicatures. 


\section{Bibliography}

van Benthem, J. (1986). Essays in Logical Semantics. Kluwer.

van den Berg, M. (1996). Some Aspects of the Internal Structure of Discourse.

The Dynamics of Nominal Anaphora. PhD dissertation, Univ. of Amsterdam.

Brasoveanu, A. (2008). Donkey Pluralities. In Linguistics and Philosophy 31, 129-209.

Büring, D. (2008). The Least at least Can Do. In Proceedings of WCCFL 26, C.B. Chang \& H.J. Haynie (eds.), Cascadilla, 114-120.

Champollion, L. (2009). Cumulative Readings of Every Do Not Provide Evidence for Events and Thematic roles. In Proceedings of $A C 1 \%$.

Chierchia, G., D. Fox \& B. Spector (to appear). The Grammatical View of Scalar Implicatures. In Handbook of Semantics, P. Portner et al (eds.), de Gruyter.

Constant, N. (2006). English Rise-Fall-Rise: A study in the Semantics and Pragmatics of Intonation. MA thesis, UC Santa Cruz.

Farkas, D.F. (2009). Varieties of Indefinites. In Proceedings of SALT XII, B. Jackson (ed.), CLC, 59-83.

Ferreira, M. (2007). Scope Splitting and Cumulativity. In Proceedings of the ESSLLI Workshop on Quantifier Modification, R. Nouwen \& J. Dotlačil (eds.).

Geurts, B. \& R. Nouwen (2007). At Least et al: The Semantics of Scalar Modifiers. In Language 83, 533-559.

Heim, I. (1982). The Semantics of Definite and Indefinite Noun Phrases, PhD dissertation, UMass. Published in 1988 by Garland.

Kratzer, A. (2000). The Event Argument and the Semantics of Verbs. UMass. ms., available at semanticsarchive.net.

Krifka, M. (1999). At least Some Determiners Aren't Determiners. In The Semantics/Pragmatics Interface from Different Points of View, K. Turner (ed.), Elsevier, 257-291.

Landman, F. (2000). Events and Plurality, Kluwer.

Lauer, S. (2009). Free relatives with -ever: Meaning and Use. Stanford, ms.

Lewis, D. (1975). Adverbs of Quantification, in Formal Semantics of Natural Language, E. Keenan (ed.), Cambridge University Press, 3-15.

Nouwen, R. (2009). Two Kinds of Modified Numerals. To appear in Semantics and Pragmatics.

Robaldo, L. (2009). Distributivity, Collectivity and Cumulativity in terms of (In)dependence and Maximality. Univ. of Turin, ms.

Roberts, C. (1989). Modal Subordination and Pronominal Anaphora in Discourse. In Linguistics and Philosophy 12, 683-721.

Schein, B. (1993). Plurals and Events. MIT Press.

Schwarzschild, R. (1996). Pluralities. Kluwer.

Stalnaker, R. (1978). Assertion, in Syntax and Semantics 9, 315-332.

Szabolcsi, A. (1997). Strategies for Scope Taking. In Ways of Scope Taking, A. Szabolcsi (ed.), Kluwer, 109-154.

Umbach, C. (2006). Why do modified numerals resist a referential interpretation? In Proceedings of SALT XV, CLC, 258-275. 